Experimental Study

\title{
e Enhanced Pain Sensitivity with Systemic Ultrastructural Changes of the Nervous Systems after Cobra Venom Injection is Reversed by Electroacupuncture Treatment
}

Guo-fang Lu, MD', Jun-ming Zhang, MD², Jian-xiong An, MD, PhD'1, Xiao-yan Qian, CRNA ${ }^{1}$, Yong Wang, MD', Si-yu Lin, MD', and John P. Williams, MD

\begin{abstract}
From: ${ }^{1}$ Aviation General Hospital, China Medical University, China; 2University of Cincinnati College of Medicine, Cincinnati, $\mathrm{OH}$; ${ }^{3} U$ niversity of Pittsburgh School of Medicine, Pittsburg, PA
\end{abstract}

Address Correspondence: Jian-xiong An, MD, PhD Aviation General Hospital China Medical University Anesthesiology, Pain Medicine \& Critical Care Medicine Beiyuan Rd 3,

Beijing, 100012, China E-mail: anjianxiong@ yahoo.com

Disclaimer: There was no external funding in the preparation of this manuscript.

Conflict of interest:

Each author certifies

that he or she, or a member of his or her immediate family, has no commercial association

(i.e., consultancies, stock ownership, equity interest, patent/licensing arrangements, etc.) that might pose a conflict of

interest in connection

with the submitted manuscript.

Manuscript received: 11-11-2017

Revised manuscript received: 02-01-2018 Accepted for publication: 03-08-2018

Free full manuscript: www. painphysicianjournal.com
Background: Electroacupuncture (EA) has been proved to be effective in treating certain neuropathic pain conditions. The mechanisms of pain relief by EA are not fully understood. There have been sporadic reports of damage in the peripheral nervous system (PNS) and regions of the central nervous system (CNS) at the ultrastructural level following peripheral nerve injury. However, information about possible systemic changes in the PNS and CNS after nerve injury is scarce.

Objectives: The goal of this study was to examine the ultrastructural changes of the nervous system induced by a local injection of cobra venom into the sciatic nerve and to compare the ultrastructural changes in rats with or without treatment with EA or pregabalin.

Study Design: An experimental study.

Setting: Department of Anesthesiology, Pain Medicine, and Critical Care Medicine, Aviation General Hospital of China Medical University.

Methods: In this study, using an established model of sciatic neuralgia induced by local injection of cobra venom into the sciatic nerve, we examined ultrastructural changes of the PNS and CNS and how they respond to EA and pregabalin treatment. EA and pregabalin were given daily from postoperative day (POD) 14 to 36. Based on previous works, the frequency of EA stimulation of the ST36 and GB34 acupoints was held to $2 / 100 \mathrm{~Hz}$ variable. Pain sensitivity in the sciatic neuralgia rats with and without treatments was assessed using the von Frey test. Ultrastructural alterations were examined bilaterally in the prefrontal cortex, hippocampus, medulla oblongata; and the cervical, thoracic, and lumbar spinal cords on PODs 14, 40, and 60. Ultrastructural examinations were also carried out on the bilateral sciatic nerves and dorsal root ganglion (DRG) at the cervical, thoracic and lumbar levels. In rats treated with EA or pregabalin, the ultrastructure was examined on PODs 40 and 60.

Results: Behavioral signs of pain and systemic ultrastructural changes including demyelination were observed at all levels of the PNS and CNS in rats with sciatic neuralgia. After intervention, the mechanical withdrawal thresholds of the EA group and pregabalin group were significantly higher than that of the cobra venom group $(P<0.05)$. Both EA and pregabalin treatments partially reversed increased cutaneous sensitivity to mechanical stimulation. However, only the EA treatment was able to repair the ultrastructural damages caused by cobra venom.

Limitations: The results confirm that peripheral nerve injury led to the ultrastructural damage at different levels of the CNS as demonstrated with electron microscopy; however, we need to further verify this at both the molecular level and in light microscope level. Sciatic neuralgia induced by cobra venom is a chemical injury, and whether this exactly mimics a peripheral nerve mechanical injury is still unclear.

Conclusions: Local cobra venom injection leads to systemic neurotoxicity. EA and pregabalin alleviate pain via different mechanisms.

Key words: Sciatic neuralgia, cobra venom, demyelination, electroacupuncture, pregabalin, rat model

Pain Physician 2018: 21:E509-E521 
$\mathrm{N}$

europathic pain is characterized by pain arising as a direct consequence of a lesion or disease affecting the somatosensory system (1). Although poorly managed, neuropathic pain has been found to be responsive to electro-acupuncture (EA) therapy as well as pregabalin treatment $(2,3)$ in humans and animal models $(4,5)$. In addition to neuropathic pain treatment, EA also protects the ultrastructure of muscles (6), neurons (7), and myelin sheath (8), and thus may promote tissue repair and nerve regeneration after injury.

As an anti-convulsant with a superior pharmacokinetic profile to its analogue gabapentin (9), pregabalin has quickly become the first-line treatment for neuropathic pain including sciatic neuralgia $(10,11)$. Similar analgesic effects of pregabalin were found in animal models of pain $(12,13)$, although its underlying mechanism is still not clear.

We recently developed an experimental model of sciatic neuralgia by injecting a small amount of cobra venom (CV) into the unilateral sciatic nerve (14). Rats that received snake venom injections quickly developed signs of sciatic neuralgia with swollen ipsilateral paws, spontaneous pain, and significantly increased mechanical sensitivity.

In another experiment, we observed widespread demyelination in the hippocampus and medulla oblongata in rats with experimental trigeminal neuralgia induced by the injection of CV into the infraorbital nerve (15). Moreover, snake venom injections resulted in Schwann cell degeneration and demyelination in the brachial plexus and the cervical spinal cord (C8-T1) (16).

In the present study, we examined systemic ultrastructural changes of the PNS and CNS in rats with sciatic neuralgia. The effects of EA therapy in pain relief were assessed and compared to that of pregabalin treatment. Finally, to explore possible mechanisms of EA and pregabalin-induced pain relief, we also compared the ultrastructural changes in rats with or without treatment with EA or pregabalin.

\section{Methods}

\section{Animals}

A total of 44 adult male Sprague-Dawley rats (250-300 g in weight) were provided by the Laboratory Animal Center of the Academy of Military Medical Sciences. Animal protocols were approved by The Animal Care and Use Committee (Beijing, China). The entire experimental procedure on conscious animals was con- sistent with the guidelines of the International Association for the Study of Pain (17). Rats were allowed free access to food and water and were kept under a light/ dark cycle (12 hours/12 hours) with an ambient temperature of $22-24^{\circ} \mathrm{C}$ and relative humidity of $40-60 \%$. Rats were randomly assigned into 4 groups: sham-operated group $(n=12), C V$ group $(n=12), C V+$ EA group $(n=$ $10)$, and $C V+$ pregabalin group $(n=10)$. Every attempt was made to minimize the number of animals used and their suffering.

\section{Rat Model of Sciatic Neuralgia}

Rats were anesthetized by intraperitoneal injection of $4 \%$ chloral hydrate $(0.8 \mathrm{~mL} / 100 \mathrm{~g})$. The biceps femoris muscle was separated by blunt dissection to expose the sciatic nerve at mid-thigh level. The nerve trunk proximal to the sciatic nerve trifurcation, about $10 \mathrm{~mm}$, was freed of interstitial tissues. Cobra venom (0.5 mg in $5 \mu \mathrm{l}$, Venom Research Institute of Guangxi Medical University, Guangxi, China) was injected into the nerve sheath using a $10 \mu \mathrm{l}$ microinjector (Shanghai Gaoge Industry, Shanghai, China). The needle was kept in place for about 5 minutes after the injection to prevent venom leakage. Rats who receive sham operations underwent a similar procedure and saline injection. The incision was closed in layers. Behavioral measurements of pain sensitivity were performed 24 hours after the initial injection.

\section{Electro-acupuncture Therapy}

EA treatment was performed according to a novel, unrestrained, unsedated, and conscious protocol originally described by Lao et al (18). After cleaning the skin with alcohol swabs, the investigator swiftly inserted disposable acupuncture needles (gauge \#32, 0.5 in. in length) into the right hindpaw at the equivalent anatomical landmarks of human "zusanli" (ST36) and "Yanglingquan" (GB34) acupoints. In humans, ST36 is located at one-finger breadth below and anterior to the tibial tuberosity (19). GB34 is located near the knee joint anterior and inferior to the small head of the fibula in the muscle peroneus longus and brevis (20). The entire procedure lasted less than 20 seconds and caused little distress to the animal. The animals were placed in a small transparent plastic cage to receive EA stimulation using a Han's Acupoint Nerve Stimulator (HANS, LH series, Peking University, Beijing, China). The frequency of EA stimulation was $2 / 100 \mathrm{~Hz}$ variable. The current intensity was kept at $1 \mathrm{~mA}$ for 10 minutes, then increased to $1.5 \mathrm{~mA}$ for another 10 minutes and 
finally $2 \mathrm{~mA}$ for 10 minutes. Each trial took 30 minutes to finish. The electrical stimulation was delivered to the under-skin muscles but not directly to the skin or the peripheral nerve, and only caused mild muscle twitching during stimulation. If the needles dropped during EA, they were reinserted as quickly as possible. In order to avoid possible tolerance, EA intervention was performed once every 3 days, from POD 14 to POD 36.

\section{Pregabalin Treatment}

Pregabalin was dissolved in $0.9 \%$ saline and administered by oral gavage using a self-made instrument. Pregabalin was previously shown to attenuate nociceptive responses following chronic constrictive injury $(\mathrm{CCl})$ of the sciatic nerve in a dose-dependent manner (3-30 $\mathrm{mg} / \mathrm{kg}$ orally) (21). In the current study, rats were given pregabalin $30 \mathrm{mg} / \mathrm{kg}$ once daily for 22 days from POD 14 to POD 36.

\section{Behavioral Assessments}

In order to avoid potential bias, the person performing the behavioral testing was blinded as to the experimental groups. To examine the analgesic effects of EA and pregabalin, behavioral testing was conducted 3 days before the injection and 3, 6, 9, 12, 15, 20, 25, 30,40 and 60 days after the initial venom injection. The testing was performed during daylight hours (7 AM to $7 \mathrm{PM}$ ). In this experiment, the mechanical withdrawal thresholds (MWTs) of the rats were determined by the up-down method (22). Briefly, the rats were placed in a clear plastic chamber $(20 \times 20 \times 20 \mathrm{~cm})$ on the surface of an elevated wire mesh floor $(1.0 \times 1.0 \mathrm{~cm})$, which allowed full access to the paws by the experimenter. A series of calibrated von Frey hairs (Stoelting, Chicago, IL) with stiffness between $0.41 \mathrm{~g}$ to $15.10 \mathrm{~g}$ were applied perpendicularly to the mid-plantar surface of both forelimb and the contralateral hind paw with adequate force to cause mild bending against the paws. A sharp withdrawal or flinch of the hind paw was considered a positive response. Pain sensitivity was not assessed on the ipsilateral hind paw due to the changes in gait and posture after venom injection (see Results).

\section{Electron Microscopic Analysis}

Two rats from each group (the sham-operated group, CV group, CV + EA group and CV + pregabalin group) were sacrificed for a transmission electron microscopic (TEM, Hitachi H-7650 NAR, Ibaraki, Japan) exami- nation on day 14,40 , and 60 days after surgery. Under deep anesthesia, rats were perfused intracardially with saline followed by a mixed solution of $4 \%$ paraformaldehyde and $2 \%$ glutaraldehyde (Sigma, St. Louis, MO). The bilateral prefrontal cortex and hippocampus; the ipsilateral medulla oblongata; the ipsilateral spinal dorsal horn and bilateral dorsal root ganglion (DRG) at cervical (C4), thoracic (T7), and lumbar (L4) levels; and the bilateral sciatic nerves were collected for analysis. Tissues were post-fixed overnight in 3\% glutaraldehyde and rinsed with $0.1 \mathrm{M}$ PB 3 times followed by fixation in $1 \%$ osmium tetroxide (Sigma, St. Louis, MO, USA) for 2 hours. Tissues were dehydrated, embedded in araldite for one day, and cut into $1 \mu \mathrm{m}$ ultrathin sections (50-70 $\mathrm{nm})$. Sections were stained with uranile acetate and observed under EM. Studies from each group were labeled with numbers so that the persons performing the image analysis were blinded.

\section{Statistical Analysis}

Statistical analyses were performed using SPSS Version 21.0 (IBM Corporation, Armonk, NY). One-way ANOVA was used for group comparisons. Within-group differences across time were evaluated by ANOVA. All data are expressed as mean \pm standard deviation (SD). A $P$ value of $<0.05$ was considered as having reached statistical significance.

\section{Results}

\section{General Observations}

All rats survived after the venom injection and were able to drink and urinate normally. Immediately after the venom injections, the ipsilateral hind paws showed signs of spontaneous pain, i.e., licking and flinching as observed in the formalin model. Beginning on day 2 after injection, the rats began to hold the affected paws above the floor while standing or walking with the toes together and ventroflexed. The gait and posture changes persisted and the rats never recovered normal gaits during the entire testing period. Autotomy was observed in about one third of the rats in the ipsilateral hind paws that received intraneural injections of CV. Prolonged treatment with EA or pregabalin did not improve the gait or posture. Mechanical sensitivity was not assessed in the ipsilateral hind paws due to gait and posture changes. The sham-operated group of rats did not show any gait changes or signs of pain. 


\section{Sciatic Nerve Venom Injection Induced Behavioral Signs of Pain in Bilateral Forepaws and the Ipsilateral Hind Paw}

As shown in Fig. 1, there were no significant differences between each group prior to venom injection. All animals were analyzed in each group (44/44). The MWTs on both forepaws and contralateral hind paw of the CV group showed remarkable decreases after injection as compared to the sham-operated group (\#P $=0.001$ ). EA treatment beginning on POD 14 decreased mechanical sensitivity to von Frey stimulation on both forepaws and the right hind paw. Significant recovery was first observed after EA treatment for 7 days $(+P=$ 0.003). The effects lasted through the testing period. A similar effect was observed in the pregabalin-treated group ( ${ }^{*} P=0.02$ ). The data suggested that both EA and pregabalin treatments were able to ameliorate neuropathic pain in the rats with sciatic neuralgia.

\section{Snake Venom Injection Caused Bilateral Ultrastructural Changes in the Sciatic Nerve}

In the sham-operated group, complete and clearly visible myelin sheaths were observed under EM on the ipsilateral (Figs. 2A, 2B, 2C) and contralateral (Figs. 2a, $2 b, 2 c)$ sciatic nerve on postoperative days 14,40 , and 60. Compared to sham-operated rats, the myelin sheath in rats with venom injection was destroyed and the surrounding tissues were dissolved on the ipsilateral (Figs. 2D, 2E, 2F) sciatic nerve. Similar changes were observed in the contralateral sciatic nerve (Figs. 2d, 2e, $2 \mathrm{f})$ although the damages appeared to be less severe compared to the ipsilateral side.

\section{Ultrastructural Changes in Brain Prefrontal Cortex and Hippocampus after Local CV Injection in the Unilateral Sciatic Nerve}

In the sham-operated rats, normal cell structures
A

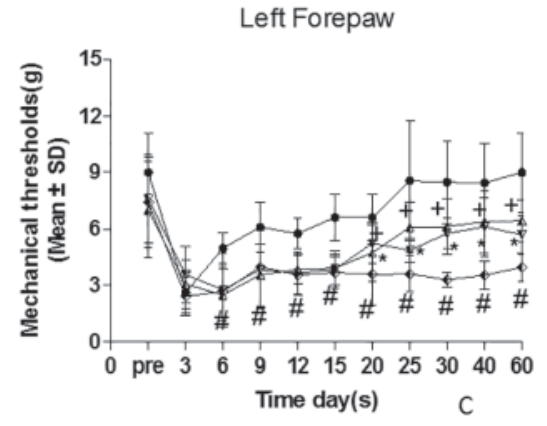

- sham-operated

- cobra venom

\pm C.V.+EA

$\rightarrow$ C.V.tpregabalin
B

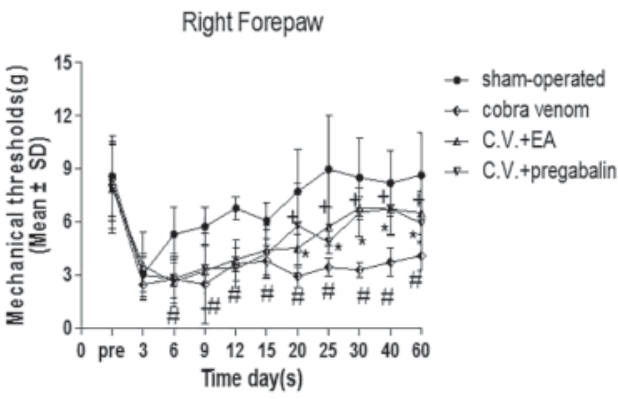

Right Hindpaw

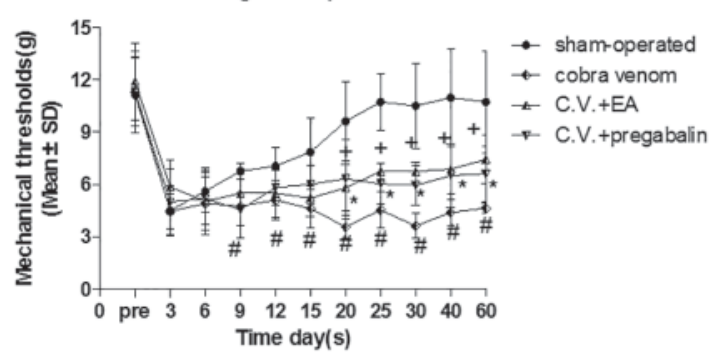

Fig. 1. Enhanced mechanical sensitivity was partially reversed by $E A$ or pregabalin treatment.

Changes in MWT were compared between groups for left forepaw (A), right forepaw (B) and right hind paw (C). There were no significant differences in the MWTs prior to treatments $(P=0.087)$. The MWT of the group injected with cobra venom showed a remarkable decrease after operation in comparison to the sham-operated group (\#: compared between rates injected with cobra venom and the shamoperation group, $\# P=0.001$ ). For the EA and pregabalin groups, the MWT showed a distinct increase after EA and pregabalin intervention $\left(+,{ }^{*}\right.$ :compared between EA and pregabalin with cobra venom, $\left.+,{ }^{*} P=0.003, P=0.02\right)$. Error bars indicate the standard deviation. Data are presented as mean $\pm \mathrm{SD}, \#,+,{ }^{\star} P<0.05$. 

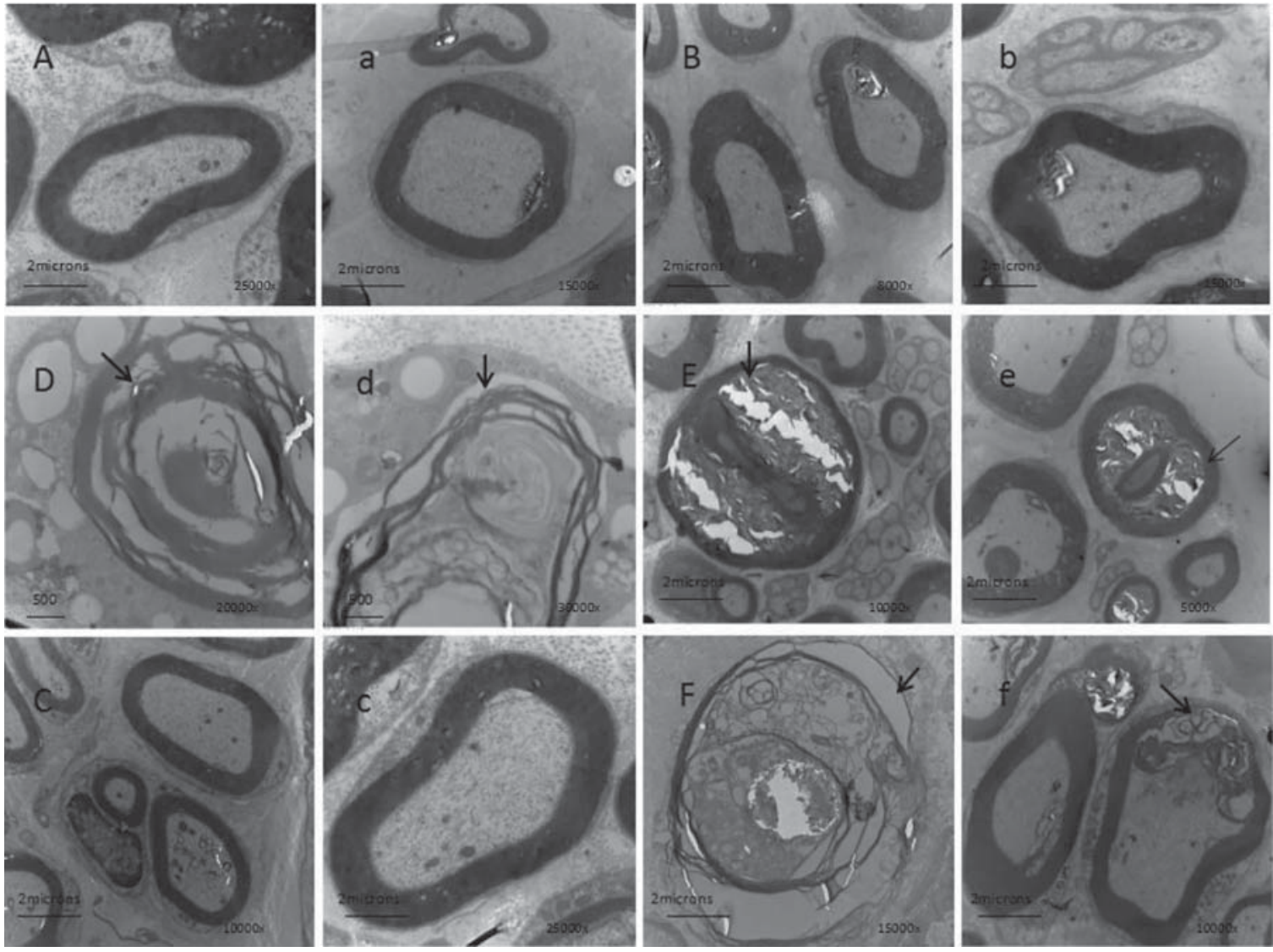

Fig. 2. Ultrastructural changes in the sciatic nerve in the cobra venom-injected rats and the sham-operation rats.

Electron microscopy was analyzed on tissues collected on POD 14, 40, and 60. A, B, C, a, b, c: completely intact myelin sheaths in the sciatic nerves in the sham-operated rats. D, E, F: Myelin sheaths were thinned or destroyed in the sciatic nerves exposed to cobra venom (marked by arrows). d, e, f: myelin on the contralateral sciatic nerve appeared to be intact (marked by arrows).

were observed with clear presynaptic membranes, post synaptic membranes, synaptic vesicles, and normal myelination on both the ipsilateral (Figs. 3A, 3C, $3 \mathrm{E}$ ) and contralateral (Fisg. 3a, 3c, 3e) prefrontal cortex and the ipsilateral (Figs. 3B, 3D, 3F) and contralateral hippocampus (Figs. 3b, 3d, 3f). In rats with venom injection, the EM observations of the prefrontal cortex found deformed cells with shrunken organelles with extensive cell vacuolar degeneration on both ipsilateral (Figs. 3G, 3I, 3K) and contralateral (Figs. 3g, 3i, $3 \mathrm{k}$ ) sides. Similar changes were observed in the ipsilateral (Figs. 3H, 3J, 3L) and contralateral (Figs. 3h, 3j, 3l) hippocampus.

\section{Snake Venom Injection of the Unilateral Sciatic Nerve Caused Ultrastructural Changes at Different Levels of the Spinal Cord}

As expected, EM observation revealed no abnormal changes in the myelination or the cellular structure in the medulla oblongata and the cervical, thoracic, and lumbar spinal dorsal horns in the sham-operated rats on POD 14 (Figs. 4A, 4B, 4C, 4D), 40 (Fig. 4E, 4F, 4G, 4H), or 60 (Figs. 4I, 4J, 4K, 4L). Conversely, the venom injections caused robust damages to the spinal cord at all levels examined. The myelin sheaths were swollen, distorted, and demyelinated on POD 14 (Figs. 4M, 4N, 4O,4P), 40 (Fig. 4Q, 4R, 4S, 4T), and 60 (Figs. 4U, 4V, 4W, 4X). 


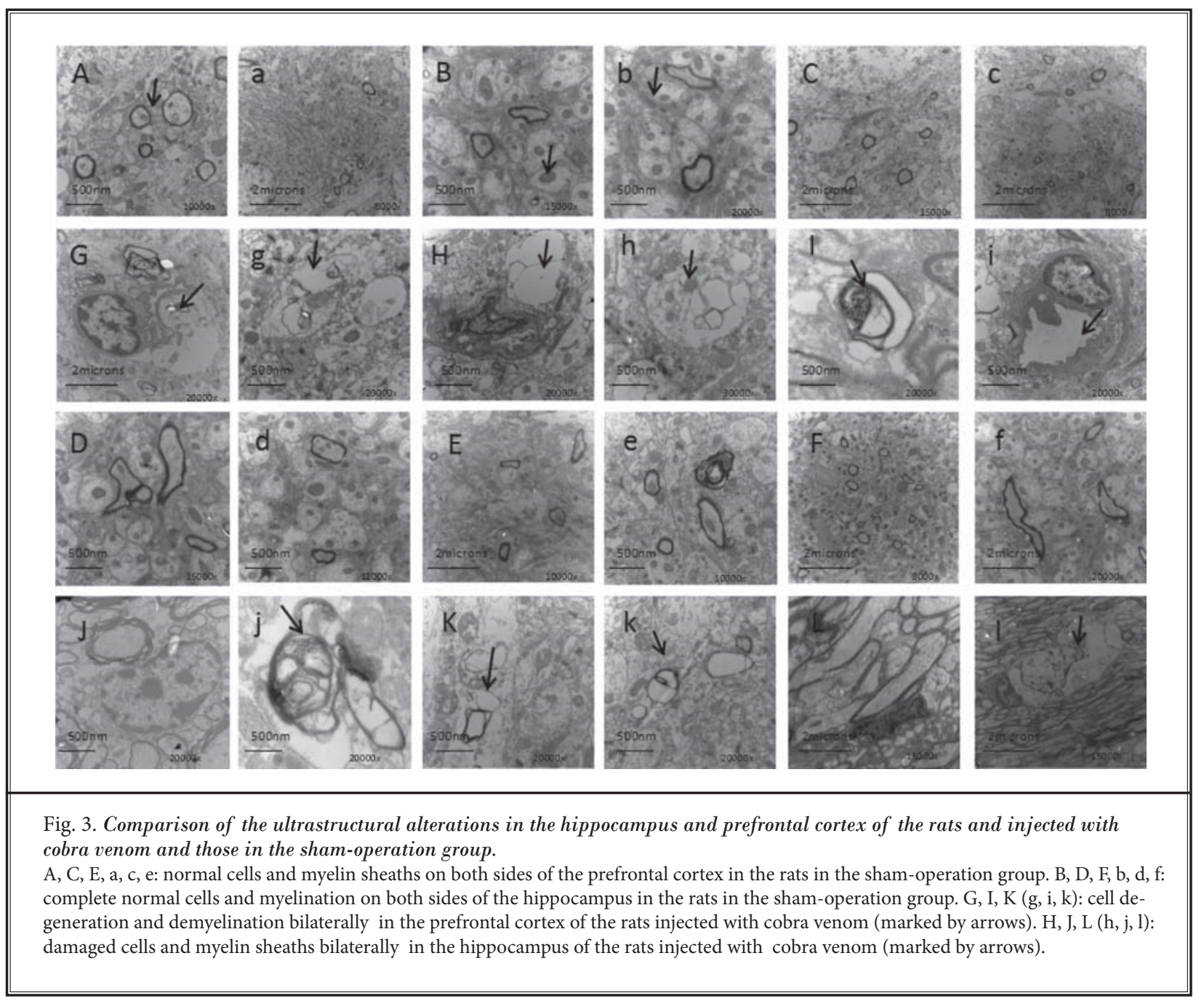

\section{Local CV Injection Resulted in Bilateral Demyelination in the DRG}

EM observations demonstrated very mild demyelination in the ipsilateral (Figs. 5A, 5B, 5C, 5D, 5E, 5F, $5 \mathrm{G}, 5 \mathrm{H}, 5 \mathrm{I}$ ) and contralateral (Figs. 5a, 5b, 5c, 5d, 5e, 5f, $5 \mathrm{~g}, 5 \mathrm{~h}, 5 \mathrm{i}) \mathrm{DRG}$ at cervical, thoracic, and lumbar levels in the sham-operated rats on POD 14 (Figs. 5A, 5B, 5C, $5 a, 5 b, 5 c)$. Normal structures or no abnormal changes were observed on POD 40 (Figs. 5D, 5E, 5F, 5d, 5e, 5f) or POD 60 (Figs.5G, 5H, 5l, 5g, 5h, 5i). In rats with venom injection, the vast majority of myelin sheaths and deformed cells were severely disintegrated on both the ipsilateral (Figs. 5J, 5K, 5L, 5M, 5N, 5O, 5P, 5Q, 5R) and contralateral (Figs. 5j, 5k, 5l, 5m, 5n, 5o, 5p, 5q, 5r) DRGs at all examined levels on POD 14 (Figs. 5J, 5K, 5L, 5j, 5k,
5I), 40 (Figs. 5M, 5N, 5O, 5m, 5n, 5o), and POD 60 (Figs. $5 P, 5 Q, 5 R, 5 p, 5 q, 5 r)$.

\section{Recovery of the Damaged Sciatic Nerve in the EA-treated Rats but not the Pregabalin- treated Ones}

Rats that received venom injections and were under EA therapy for 22 days showed significant recovery in the damaged sciatic nerve. beginning on POD 14 Only mild swelling of the myelin sheath was observed under electron microscopy in both the ipsilateral and contralateral sides on POD 40 and 60 (Figs. 6A, 6B). Rats that received venom injections and received pregabalin treatment, however, did not show any improvement. Dissolved myelin sheaths were still visible in the ipsilateral and contralateral sciatic nerves on POD 40 and 60 


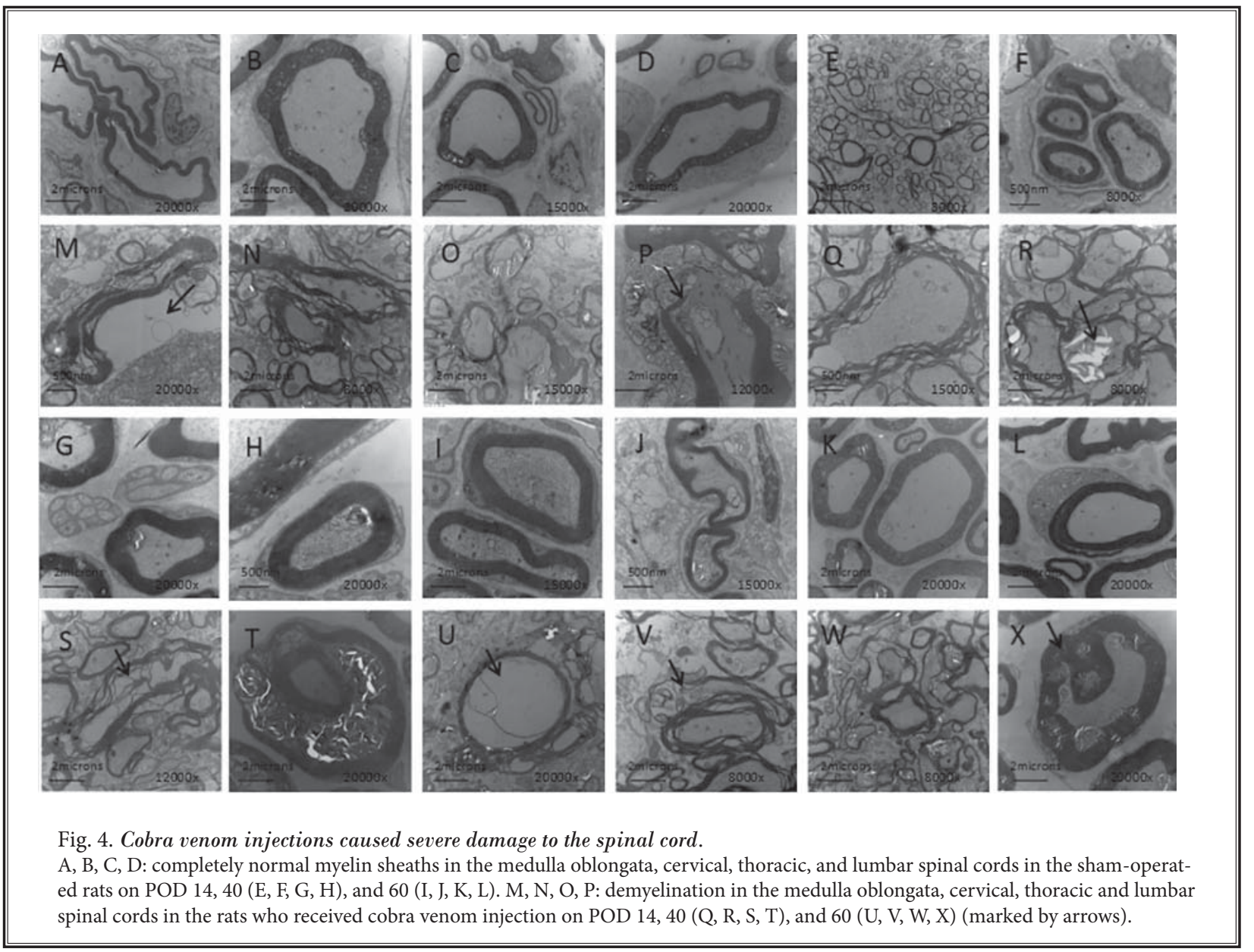

(Figs. 6C, 6D). Some of the ultrastructure changes are shown in Table 1.

\section{EA-treatment Restored Normal Structures of the Spinal Cord, the Prefrontal Cortex, and the Hippocampus}

In addition to the sciatic nerve, we also observed restored structures at all levels of the CNS after EA treatment. Normal-shaped cells with dendrites and intact myelin sheaths could be seen on the ipsilateral and contralateral sides of the brain prefrontal cortex and hippocampus on POD 40 and 60 (Fig. 7A, 7B). The damaged spinal cord including the medulla oblongata also showed robust improvement after EA treatment on POD 40 and POD 60, as shown in Fig. 7C; but not after treatment with pregabalin, which still showed deformed cells, shrunken nucleus and organelles, and severe cell vacuoles degeneration on the ipsilateral and contralateral prefrontal cortex and hippocampus (Fig. 7D, 7E). Pregabalin treatment had no effect on the ultrastructural changes that occurred in the spinal cord on POD 40 or 60 (Fig. 7F).

\section{EA but not Pregabalin Treatment Reversed Ultrastructural Slterations in DRGs after Local Venom Injection}

EM observation showed complete and normal myelin sheaths on the ipsilateral and contralateral DRGs at all vertebral levels in EA-treated rats as observed on POD 40 and 60 (Figs. 8A, 8B). In the pregabalin-treated rats, however, partial medullary dissolution and alteration of onion-like demyelination were shown on the ipsilateral and contralateral DRGs at all levels (Fig. 8C, 8D). 


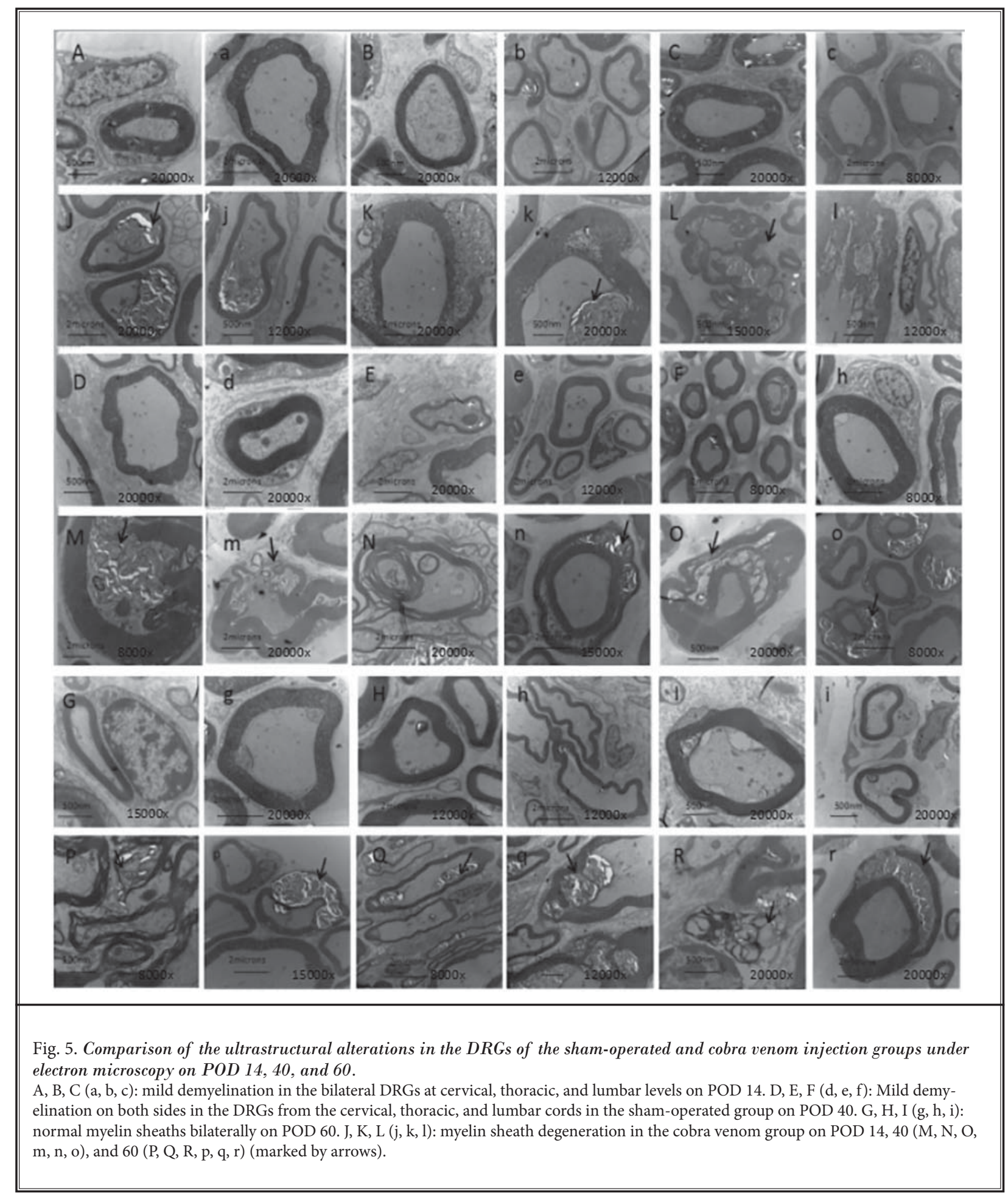


Fig. 6. Comparison of the ultrastructural alterations in the sciatic nerve of the $E A$ and pregabalin groups under electron microscopy on POD 40, 60.

A, B: mild demyelination of the sciatic nerve in the EA group. C, D: vacuolar degeneration and swollen myelin sheaths in the sciatic nerves in the pregabalin group (marked by arrows).
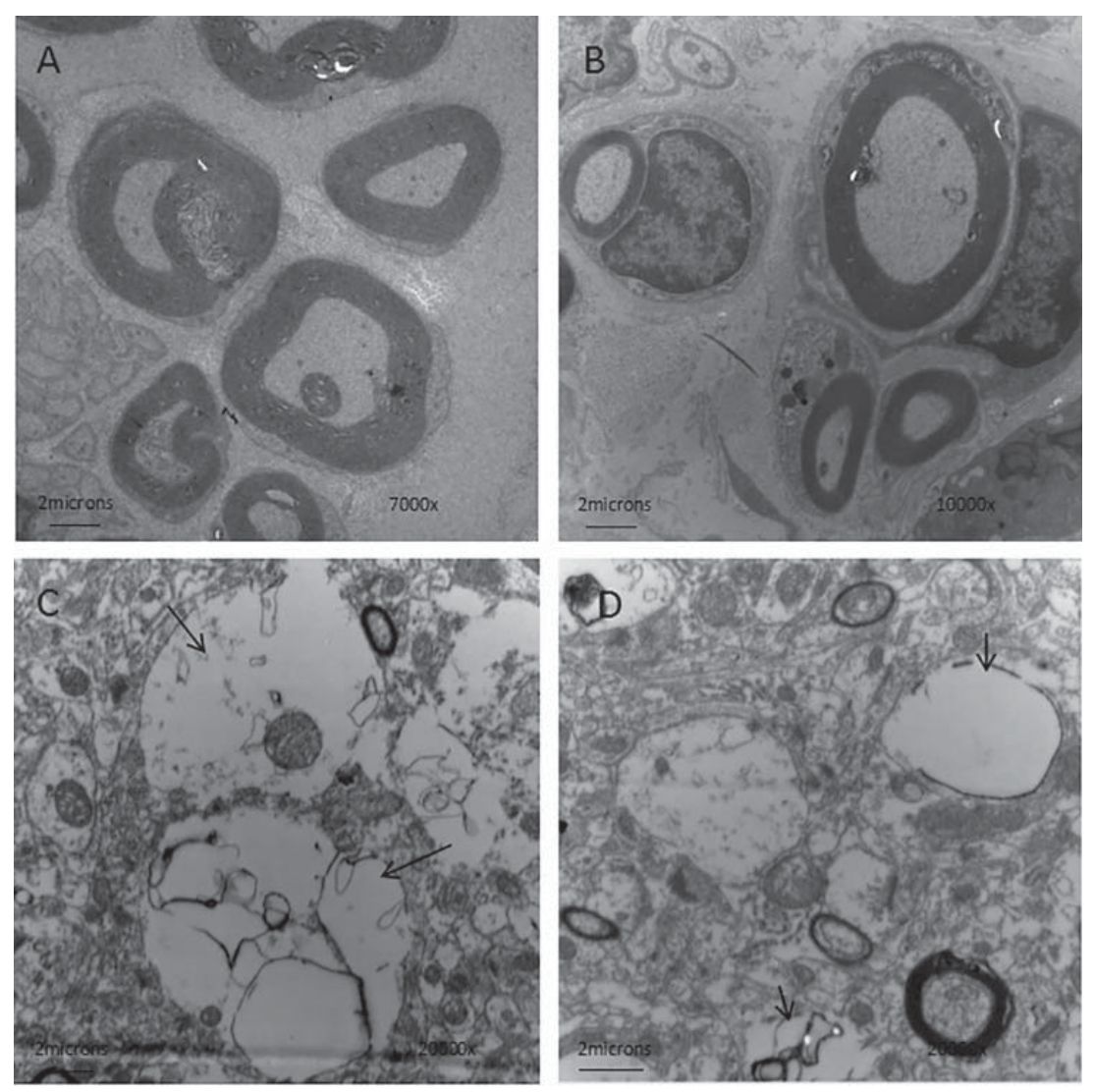

\section{Discussion}

Commonly used animal models of neuropathic pain including the chronic constriction injury model (CCI) (23), the partial sciatic nerve injury model (PNL) (24), the spinal nerve ligation model (SNL) (25), and the spared nerve injury model (SNI) (26) are developed by partial or incomplete nerve injury. These models are useful in the study of peripheral neuropathy that is limited to a single extremity. Clinically, many neuropathic pain conditions have impact on multiple limbs and sometimes even the whole body. Examples include diabetic neuropathy, chemotherapy-induced peripheral neuropathy, and pain syndromes resulting from autoimmune diseases.

In all rats that received CV injection in the sciatic nerve, the ipsilateral hind paw appeared swollen, and several of the rats showed autotomy as early as the third day after venom injection. Further, the MWTs of the right hind paw and both forepaws demonstrated mechanical hypersensitivity. In addition, when compared to the sham-operated group, the ultrastructure at different levels of the nervous system in the CV group was severely damaged. We were not able to measure mechanical sensitivity of the ipsilateral hind paw because of changes in gait and posture. However, the presence of mechanical allodynia in the contralateral paw and the forepaws suggests the development of whole-body allodynia, which may have been caused by damage beyond the sciatic nerve. $\mathrm{CV}$ is known to be neurotoxic and the widespread ultrastructural changes in the PNS and CNS may have been caused by systemic neurotoxicity of the venom diffused in the circulatory system. Zhu et al (14) showed that if venom is put outside of the nerve instead of into the nerve, the rat dies. Thus, the diffusion of the venom is somewhat limited with intraneural injections. In addition, the observed systemic ultrastructural changes in the current study 
Pain Physician: September/October 2018: 21:E509-E521

Table 1. A summary of the ultrastructural changes in the PNS and CNS with and without EA or pregabalin treatment.

\begin{tabular}{|l|c|c|c|c|}
\hline \multirow{2}{*}{ PNS and CNS } & \multicolumn{2}{|c|}{ POD 14, 40, 60 } & \multicolumn{2}{c|}{ POD 40, 60 } \\
\cline { 2 - 5 } & Sham-operated & C.V. & C.V.+ EA & C.V.+ Pregabalin \\
\hline $\begin{array}{l}\text { Bilateral prefrontal cortex } \\
\text { and hippocampus }\end{array}$ & $\begin{array}{c}\text { Normal cellular structure, } \\
\text { normal pre- and post- } \\
\text { synaptic membranes, } \\
\text { normal vesicles, and } \\
\text { normal myelination }\end{array}$ & $\begin{array}{c}\text { Shrunken organelles, } \\
\text { deformed cell, and } \\
\text { demyelination }\end{array}$ & $\begin{array}{c}\text { Normal-shaped cells and } \\
\text { intact myelin sheaths }\end{array}$ & $\begin{array}{c}\text { Deformed cells, shrunken } \\
\text { nucleus, organelles, and } \\
\text { severe degeneration of cell } \\
\text { vacuoles }\end{array}$ \\
\hline $\begin{array}{l}\text { Ipsilateral spinal dorsal } \\
\text { horn }\end{array}$ & $\begin{array}{c}\text { Normal structure of cell } \\
\text { and myelin sheaths }\end{array}$ & $\begin{array}{c}\text { Swollen, distorted myelin } \\
\text { sheaths and demyelination }\end{array}$ & Slight demyelination & Severe demyelination \\
\hline $\begin{array}{l}\text { Bilateral DRG at cervical, } \\
\text { and thoracic, lumbar levels }\end{array}$ & $\begin{array}{c}\text { Mild demyelination } \\
\text { observed within the } \\
\text { ganglia }\end{array}$ & $\begin{array}{c}\text { Disintegrated myelin } \\
\text { sheaths }\end{array}$ & $\begin{array}{c}\text { Complete and normal } \\
\text { myelin sheaths }\end{array}$ & $\begin{array}{c}\text { Medullary dissolution, } \\
\text { onion-like demyelination }\end{array}$ \\
\hline Bilateral sciatic nerve & $\begin{array}{c}\text { Complete and clear myelin } \\
\text { sheaths }\end{array}$ & $\begin{array}{c}\text { Completely dissolved } \\
\text { myelin sheaths at the } \\
\text { injection site }\end{array}$ & $\begin{array}{c}\text { Significant recovery of the } \\
\text { myelin sheaths }\end{array}$ & $\begin{array}{c}\text { Dissolved myelin sheaths } \\
\text { at the injection site }\end{array}$ \\
\hline
\end{tabular}
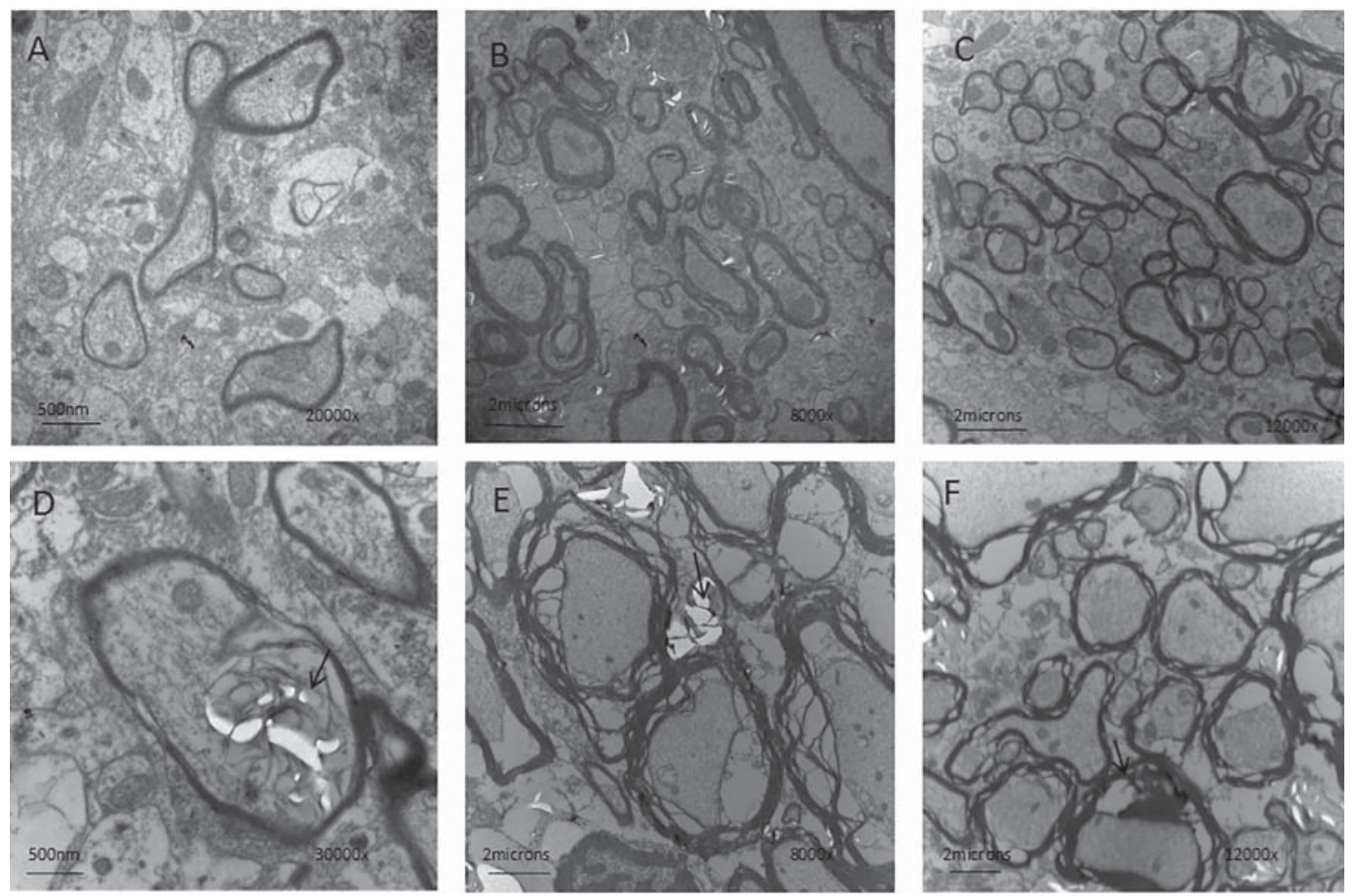

Fig. 7. Comparison of the ultrastructural alterations at various levels of the spinal cord, prefrontal cortex and hippocampus in venom-injected rats treated with $E A$ and pregabalin.

A, B: normal cells and myelin sheaths of the prefrontal cortex and hippocampus from rats treated with EA. C: mild demyelination in the spinal cord. D, E: damaged cells and extensive demyelination in prefrontal cortex and hippocampus in rats treated with pregabalin (marked by arrows). F: massive cell vacuolar degeneration and demyelination observed in the spinal cord in rats treated with pregabalin (marked by arrows). 
Fig. 8. Comparison of the ultrastructural alterations in the DRGs from rats treated with EA or pregabalin observed under electron microscopy on POD 40 and 60.

A, B: normal myelin sheaths of the DRGs in the EA group on POD 40 and 60. C, D: myelin sheath degeneration in the DRGs from pregabalin group on POD 40 and 60 (marked by arrows).
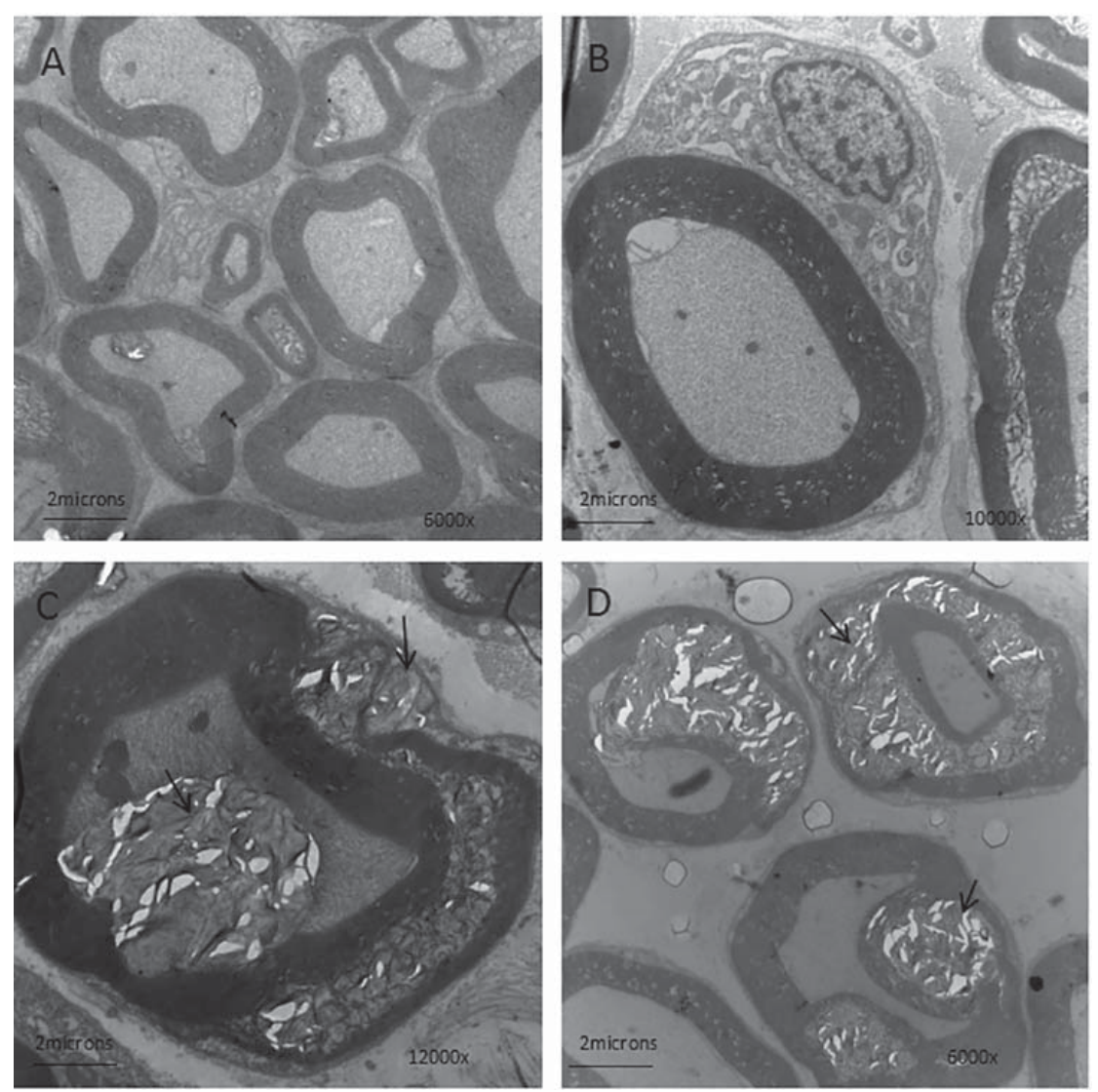

could be attributed to the higher dose of snake venom used. Previous reports by Zhu et al (14) that used lower doses of snake venom did not see demyelination, except very locally.

Considering the widespread damage of the PNS and CNS after venom injection, one would expect the animals should hardly have been able to walk around and feed themselves. Apparently, this was not the case since rats with venom injection appeared to be healthy and able to walk freely with the ipsilateral hind paw held above the floor. One possibility is that damages induced by the venom injections are limited to the sensory nervous systems in the CNS that processes pain information.

Pregabalin is generally believed to be one of the most effective anticonvulsants for treating neuropathic pain (2). Possible mechanisms underlying pregabalin's analgesic effects could be related to inhibition of the $\alpha 2 \delta$ subunit of voltage-dependent calcium channels (27) and decreased releases of substance $P$, norepinephrine, and glutamate (28).
To our surprise, EA treatment in rats with sciatic nerve venom injections not only alleviated neuropathic pain behaviors, but also significantly facilitated repair to the damaged nervous systems. Han et al (29) reported that releases of endogenous opioids play an essential role in mediating the analgesic effect of EA. They found that opioid release is frequency-dependent; EA at lower frequency (e.g., $2 \mathrm{~Hz}$ ) accelerated the release of enkephalin, beta-endorphin, and endomorphin, while higher frequency (e.g., $100 \mathrm{~Hz}$ ) selectively increased the release of dynorphin. A combination of the 2 frequencies produced a simultaneous release of all 4 opioid peptides and resulted in maximumtherapeutic effect (29-31).

Although it is not clear why EA treatment was able to facilitate the repair of the damaged nervous system, it is known that prolonged EA stimulation up-regulates neurotrophins such as nerve growth factor (NGF) while simultaneously down-regulating Fos expression (3). Our lab also found that EA treatment was able to restore the ultrastructural changes induced by the venom in- 
jections in the rat models of trigeminal and brachial plexus neuralgia $(15,16)$. Unfortunately, EA treatment failed to improve the gait changes of the ipsilateral hind paws, perhaps due to the more profound damage at the injection site.

In summary, results from the current study demonstrated that local CV injection resulted in widespread neurotoxicity, and possibly whole-body neuropathy, which is consistent with the widely increased mechanical sensitivity to mechanical stimulation. EA, but not pregabalin treatment, facilitated nerve repair caused by $\mathrm{CV}$ injection. Furthermore, in clinical studies, Inone et al (32) suggested that EA treatment has the advantage of making a deeper insertion with minimal tissue damage. Our findings are also supported by Wong et al (33), in that EA treatment may promote neurologic and functional recoveries.

\section{Conclusion}

In brief, these results indicate that CV-induced injury of the peripheral nerves leads to widespread ultrastructural damage at various levels of the nervous system. Additionally, EA treatment not only decreases neuropathic pain behavior induced by $\mathrm{CV}$, but also repairs the ultrastructural damage associated with sciatic neuralgia.

\section{Limitation}

We cannot exclude the possibility that reducing the dose of $\mathrm{CV}$ have reduced pain-related behaviors and minimized ultrastructural changes. In addition, the results confirm that peripheral nerve injury led to the ultrastructural damage at different levels of the CNS as demonstrated with electron microscopy; however, we need to further verify this at both the molecular level and in light microscope level. Sciatic nerve neuralgia induced by CV is a chemical injury, and whether this exactly mimics a peripheral nerve mechanical injury is still unclear.

\section{References}

1. Haanpaa M, Attal N, Backonja M, Baron R, Bennett M, Bouhassira D, Cruccu G, Hansson P, Haythornthwaite JA, lannetti GD, Jensen TS, Kauppila T, Nurmikko TJ, Rice AS, Rowbotham M, Serra J, Sommer C, Smith BH, Treede RD. NeuPSIG guidelines on neuropathic pain assessment. Pain 2011; 152:14-27.

2. Kumar N, Laferriere A, Yu JS, Leavitt A, Coderre TJ. Evidence that pregabalin reduces neuropathic pain by inhibiting the spinal release of glutamate.] Neurochem 2010; 113:552-561.

3. Liu YL, Li Y, Ren L, Dai LL, Bai ZH, Bai $\mathrm{R}, \mathrm{Ma}$ TM. [Effect of deep electroacupuncture stimulation of "Huantiao" (GB 30) on changes of function and nerve growth factor expression of the injured sciatic nerve in rats]. Zhen Ci Yan Jiu 2014; 39:93-99.

4. Kim HN, Park JH, Kim SK, Sun B, Koo S, Choi SM, Bae H, Min BI. Electroacupuncture potentiates the antiallodynic effect of intrathecal neostigmine in a rat model of neuropathic pain. J Physiol Sci 2008; 58:357-360.

5. Yim YK, Lee $\mathrm{H}$, Hong KE, Kim YI, Lee BR, Son CG, Kim JE. Electro-acupunc- ture at acupoint $\mathrm{ST}_{3} 6$ reduces inflammation and regulates immune activity in Collagen-Induced Arthritic Mice. Evid Based Complement Alternat Med 2007; 4:51-57.

6. Hoang NS, Sar C, Valmier J, Sieso V, Scamps F. Electro-acupuncture on functional peripheral nerve regeneration in mice: a behavioural study. BMC Complement Altern Med 2012; 12:141.

7. Wang $M$, Zhang XM, Yang SB. Effect of electroacupuncture on the expression of Glycyl-tRNA synthetase and ultrastructure changes in atrophied rat peroneus longus muscle induced by sciatic nerve injection injury. Evid Based Complement Alternat Med 2016; 2016:7536234.

8. Ding $Y$, Zhang RY, He B, Liu Z, Zhang $\mathrm{K}$, Ruan JW, Ling EA, Wu JL, Zeng YS. Combination of electroacupuncture and grafted mesenchymal stem cells overexpressing TrkC improves remyelination and function in demyelinated spinal cord of rats. SCI Rep 2015; 5:9133.

9. Guay DR. Pregabalin in neuropathic pain: A more "pharmaceutically elegant" gabapentin? Am J Geriatr Pharmacother 2005; 3:274-287.

10. Baron R, Brunnmuller U, Brasser M,
May M, Binder A. Efficacy and safety of pregabalin in patients with diabetic peripheral neuropathy or postherpetic neuralgia: Open-label, non-comparative, flexible-dose study. Eur Pain 2008; 12:850-858.

11. Moulin DE, Clark AJ, Gilron I, Ware MA, Watson CP, Sessle BJ, Coderre T, Morley-Forster PK, Stinson J, Boulanger A, Peng $P$, Finley $G A$, Taenzer $P$, Squire $P$, Dion D, Cholkan A, Gilani A, Gordon A, Henry J, Jovey R, Lynch M, Mailis-Gagnon A, Panju A, Rollman GB, Velly A, Canadian Pain S. Pharmacological management of chronic neuropathic pain - consensus statement and guidelines from the Canadian Pain Society. Pain Research \& management 2007; 12:13-21.

12. Liu C, Qian X, JianXiong AN, Wang Y, Fang Q, Jiang Y, Cope DK, Williams JP. A new animal model of brachial plexus neuralgia produced by injection of cobra venom into the lower trunk in the rat. Pain Med 2015; 16:1680-1689.

13. Zhao QQ, Qian XY, An JX, Liu CC, Fang QW, Wang Y, Jiang YD, Cope DK, Williams JP. Rat model of trigeminal neuralgia using cobra venom mimics the electron microscopy, behavioral, and anticonvulsant drug responses 
seen in patients. Pain Physician 2015; 18:E1083-E1090.

14. Zhu YL, Xie ZL, Wu YW, Duan WR, Xie YK. Early demyelination of primary Afibers induces a rapid-onset of neuropathic pain in rat. Neuroscience 2012; 200:186-198.

15. Chen RW, Liu H, An JX, Qian XY, Jiang YD, Cope DK, Williams JP, Zhang R, Sun LN. Cognitive effects of electro-acupuncture and pregabalin in a trigeminal neuralgia rat model induced by cobra venom. J Pain Res 2017; 10:1887-1897.

16. Liu H, Qian XY, An JX, Liu CC, Jiang YD, Cope DK, Williams JP. Analgesic effects and neuropathology changes of electroacupuncture on curing a rat model of brachial plexus neuralgia induced by cobra venom. Pain Physician 2016; 19:E435-E447.

17. Zimmermann M. Ethical guidelines for investigations of experimental pain in conscious animals. Pain 1983; 16:109-110.

18. Lao L, Zhang RX, Zhang G, Wang X, Berman BM, Ren K. A parametric study of electroacupuncture on persistent hyperalgesia and Fos protein expression in rats. Brain Research 2004; 1020:18-29.

19. Lin JG, Lo MW, Wen YR, Hsieh CL, Tsai SK, Sun WZ. The effect of high and low frequency electroacupuncture in pain after lower abdominal surgery. Pain 2002; 99:509-514.

20. Han P, Liu S, Zhang M, Zhao J, Wang Y,
Wu G, Mi W. Inhibition of pinal spinal interlukin-33/st2 signaling and downstream erk and jnk pathways in electroacupuncture analgesia in formalin mice. PloS One 2015; 10:e0129576.

21. Field MJ, Bramwell S, Hughes J, Singh L. Detection of static and dynamic components of mechanical allodynia in rat models of neuropathic pain: Are they signalled by distinct primary sensory neurones? Pain 1999; 83:303-311.

22. Chaplan SR, Bach FW, Pogrel JW, Chung JM, Yaksh TL. Quantitative assessment of tactile allodynia in the rat paw. J Neurosci Methods 1994; 53:55.

23. Bennett GJ, Xie YK. A peripheral mononeuropathy in rat that produces disorders of pain sensation like those seen in man. Pain 1988; 33:87-107.

24. Seltzer Z, Dubner R, Shir Y. A novel behavioral model of neuropathic pain disorders produced in rats by partial sciatic nerve injury. Pain 1990; 43:205-218.

25. Chung JM, Kim HK, Chung K. Segmental spinal nerve ligation model of neuropathic pain. Methods in Molecular Medicine 2004; 99:35-45.

26. Decosterd I, Woolf CJ. Spared nerve injury: An animal model of persistent peripheral neuropathic pain. Pain 2000; 87:149-158.

27. Houghton KT, Forrest A, Awad A, Atkinson LZ, Stockton S, Harrison PJ, Geddes JR, Cipriani A. Biological rationale and potential clinical use of gabapentin and pregabalin in bipolar disorder, insomnia and anxiety: Protocol for a systematic review and meta-analysis. BMJ Open 2017; 7:eo13433.

28. Fehrenbacher JC, Taylor CP, Vasko MR. Pregabalin and gabapentin reduce release of substance $P$ and CGRP from rat spinal tissues only after inflammation or activation of protein kinase C. Pain 2003; 105:133-141.

29. Han JS. Acupuncture and endorphins. Neurosci Let 2004; 361:258-261.

30. Huang C, Huang ZQ, Hu ZP, Jiang SZ, Li HT, Han JS, Wan Y. Electroacupuncture effects in a rat model of complete Freund's adjuvant-induced inflammatory pain: antinociceptive effects enhanced and tolerance development accelerated. Neurochem Res 2008; 33:2107-2111.

31. Liang J, Ping XJ, Li YJ, Ma YY, Wu LZ, Han JS, Cui CL. Morphine-induced conditioned place preference in rats is inhibited by electroacupuncture at 2 $\mathrm{Hz}$ : role of enkephalin in the nucleus accumbens. Neuropharmacology 2010; 58:233-240.

32. Inoue M, Hojo T, Yano T, Katsumi Y. The effects of electroacupuncture on peripheral nerve regeneration in rats. Acupunct Med 2013; 21:9-17.

33. Wong AMK, Leong CP, Su TY, Yu SW, Tsai WC, Chen CPC. Clinical trial of acupunture for patients with spinal cord injuries. Acupuncture 2003; 82:21-27. 